\title{
An Algorithm Combining Synchronization and Channel Estimation for OFDM Systems
}

\author{
Pham Hong Lien, Nguyen Duy Lai \\ Electrical and Electronics Engineering Faculty, Ton Duc Thang University, Vietnam \\ Electrical and Electronics Engineering Faculty, Ho Chi Minh City University of Transport, Vietnam \\ Email: phamhonglien2005@tut.edu.vn, lainguyenduy@hcmutrans.edu.vn
}

\begin{abstract}
OFDM (Orthogonal Frequency Division Multiplexing) is more and more popular in applications of digital communications because of the effective spectrum and less impacts of multipath fading. However, beside these advantages, OFDM signals are destroyed easily by errors such as CFO (Carrier Frequency Offset), SFO (Sampling Clock Frequency Offset). Thus, it's necessary to have robustly offset algorithms to overcome these disadvantages. Studies about OFDM we just examined channel estimation with assumptions that synchronization is perfect, and vice versa. However, they have a close relationship, channel estimation can be restricted if synchronization is bad, and vice versa. This paper presents an algorithm combining synchronization and channel estimation in OFDM systems. The algorithm is compared with other proposed algorithms by simulation. The simulation result of the algorithm combining synchronization and channel estimation is close to that of ideal conditions: perfect channel estimation and synchronization.
\end{abstract}

Keywords: Synchronization and channel estimation, OFDM, PHN (Phase Noise).

\section{INTRODUCTION}

With the incessant development of the technical science, the communication is easier and easier, better and better. Moreover, with the growing popularity of wireless networks, peoples' needs are satisfied rapidly and conveniently. Nowadays, radio networks not only transmit voice for the communication, but also support multimedia such as images, video, good quality audio, wireless internet, etc. $2.5 \mathrm{G}$ and $3 \mathrm{G}$ are being used all over the world, and $4 \mathrm{G}$ is being researched and developed. So, frequency and bandwidth must be examined to satisfy these applications. The systems are affected easily by problems such as loss transmissions in high frequency, Doppler shift in high velocities, etc. Therefore, frequency is often limited in $5 \mathrm{GHz}$ band. Besides, radio and wireless networks is more and more developed, efficient bandwidth usage is very necessary and is a challenge for researchers in the telecommunication field.

In transmission lines in broadband, beside AWGN (Additive White Gaussian Noise), signals are also affected by ISI (Inter-Symbol Interference). ISI noise is caused by delay in transmitting signals. ISI will decrease when the cycle of symbols is more than the delay of channel. So, instead of signals transmitted with high speed in a wideband channel, they can be transmitted parallel in multi-channel that have lower speed and more narrow bandwidth called subchannels. With a constant bandwidth, symbol interval will increase if the number of sub-channels increases. Then, ISI of every sub-channel will decrease significantly. This approach is called Multi-channel and OFDM is an application of the approach.

OFDM technique is based on orthogonality of subchannels. It not only helps systems save bandwidth and transmit high speed data, but also be against frequency selective fading and multipath delay. OFDM has been applied in DAB (Digital Audio Broadcasting), DVB (Digital Video Broadcasting), xDSL, IEEE 802.11a, HIPERLAN/2, and being utilized in MIMO-OFDM, MC-CDMA, WiMAX, etc. Beside its advantages, OFDM also has disadvantages affecting the received signals seriously. In OFDM, sub-channels are orthogonal together, spectra of every 
sub-channel are in form of $\sin c(f)$ function and they overlap together. However, signals are only orthogonal at the peak of $\operatorname{sinc}(f)$ function, so if there are errors in sampling, signals will have ICI (Inter Channel Interference). Moreover, as OFDM utilizes many sub-channels, there are some restrictions. The main restriction in OFDM is that it is very sensitive with synchronizing errors such as CFO and SFO. Many researchers and Labs in the world have been studying methods to eliminate these restrictions. In the first time, researches on OFDM have only examined channel and synchronization separately [2, 3, 4]. In these studies, channel estimation was done with assumptions that the synchronization is perfect $[5,6]$ and vice versa. In practice, however, channel estimation and synchronization problems are related together, channel estimation can be affected by bad synchronizations and vice versa. Therefore, there were some methods recently proposed to combine channel estimation and synchronization to each other. In [7] and [8], SFO was assumed zero, only examining CFO. On the other hand, CFO was eliminated in [9]. This paper follows the ways combining channel estimation and synchronization, and presents a robust algorithm to overcome restrictions of OFDM such as CFO, SFO and channel problems.

The paper has 5 sections: I. Introduction, II. System description, III. The Algorithm combining synchronization and channel estimation in OFDM system, IV. Simulation results, and V. Conclusion.

\section{SYSTEM DESCRIPTION}

OFDM technique is an instance of multi-carrier modulation. Binary data is modulated and becomes complex symbols. The modulation block encodes bits to become QAM/QPSK symbols. Then, the signal inserts CP (Cyclic Prefix) to decrease ISI effects.

Fig. 1 shows OFDM system. Firstly, the signal is transformed from serial to parallel and grouped to $\mathrm{x}$ bit groups to create QAM/QPSK symbols. Then, these symbols are modulated IDFT, next the signal is transformed from parallel to serial and transmitted to channel. The receiver will perform inversion comparing with the transmitter.

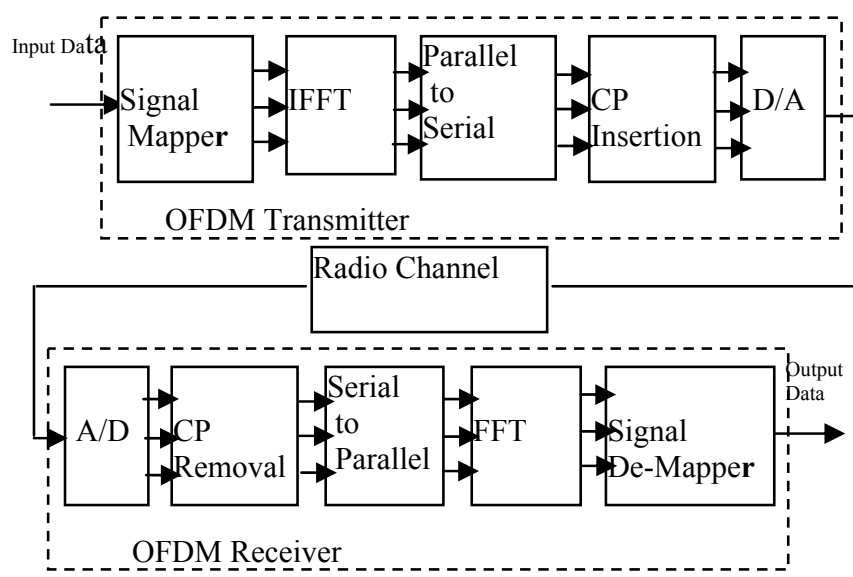

Figure 1: OFDM block diagram

Bandwidth of sub-channels in OFDM signal is $\operatorname{sinc}(f)$ forms with center frequencies $\mathrm{f}_{\mathrm{i}}=\mathrm{i} / \mathrm{T}(\mathrm{i}=$ $0,1, \ldots, M-1)$, overlapping together. These spectra will create ISI and ICI. Especially, ICI will increase if sampling errors increase. In OFDM, to decrease ISI, the transmitter has to utilize $\mathrm{CP}$ to increase the symbol interval. To decrease ICI, image channels are used.

\section{A. Mathematical fomula of the OFDM symbol}

In Fig. 1, the OFDM transmitter utilizes an M-ary modulation (M-QAM/PSK). Serial to parallel block groups bits to become Q-bit sequences, $\mathbf{d}_{1, k}$, where $\mathbf{d}_{l, k}=\left[d_{l, k}^{q}, q=0,1, \ldots, Q-1\right] \quad$ and $Q=\log _{2} M \quad$ bits. Then, mapping Q-bit, $\mathbf{d}_{l, k}$, and becoming complex symbols $X_{m}(k) \in \mathbf{A}=\left\{A_{l}, l=0,1, \ldots, M-1\right\}$, where A is modulated M-ary symbols and $m ; k$ are symbol indexes; sub-carriers indexes of OFDM symbols. Every OFDM symbol consists of $K<N$ sub-carriers bring up information, $\mathrm{N}$ is size of FFT block, $T$ is sampling cycle at output of FFT, $N_{g}$ is the number of CP sample, $T_{s}=\left(N+N_{g}\right) T$ is the symbol interval after inserting CP. After inserting $\mathrm{CP}$ and going through $\mathrm{D} / \mathrm{A}$ block, the transmitted baseband signal is given by:

$$
s(t)=\frac{1}{N} \sum_{m=-\infty}^{+\infty} \sum_{k=-K / 2}^{K / 2-1} X_{m}(k) e^{j \frac{2 \pi k}{N T}\left(t-T_{g}-m T_{s}\right)} U\left(t-m T_{s}\right),
$$


The OFDM signal is transmitted in multi-path fading channels that is given by the impulse responses as:

$$
h(t)=\sum_{i=0}^{L-1} \alpha_{i}(t) \delta(n-i)
$$

where $\alpha_{i}(t)$ is transmission gain, $\mathrm{L}$ is the number transmission lines being able to happen in the fact.

Assuming that the channel changes very slowly in time, so channel impulse responses of CIR (Channel Impulse Response), denoted by $\mathbf{h}=\left[h_{0}, h_{1}, \ldots, h_{L-1}\right]$, still unchange in the time of a transmitted data packet (burst/packet).

In the ideal case, in the receiver, after rejecting $\mathrm{CP}$, the $\mathrm{n}^{\text {th }}$ sample of the $m^{\text {th }}$ symbol of the received signal in time domain is represented by:

$$
r_{m, n}=\frac{1}{N} \sum_{k=K / 2}^{K / 2-1} X_{m}(k) H(k) e^{j \frac{2 p k}{N} n}+w_{m}\left(n+N_{m}\right)
$$

where $\quad n=0,1, \ldots, N-1 \quad$ and $\quad N_{m}=N_{g}+m\left(N+N_{g}\right)$, $w_{m}\left(n+N_{m}\right)$ is Gauss noise, they are complex values, the mean is zero and variance is $\sigma^{2} \cdot H(k)=\sum_{l=0}^{L-1} h_{l} e^{-j \frac{2 \pi k}{N} l}$ is the channel response of $\mathrm{k}^{\text {th }}$ sub-carrier. To reject ISI completely, $\mathrm{CP}$ interval must be longer than channel excess delay, L.

After transforming FFT, samples in frequency domain are $Y_{m}(k)=\sum_{n=0}^{N-1} r_{m, n} e^{-j \frac{2 \pi}{N} n k}$. From equation (3), we can show:

$$
Y_{m}(k)=\sum_{i=-K / 2}^{K / 2-1} X_{m}(i) H(i) \cdot \delta_{i, k}+W_{m}(k)
$$

where $\delta_{i k}=\frac{1}{N} \sum_{n=0}^{N-1} \mathrm{e}^{j \frac{2 \pi}{N} n(i-k)} \approx \operatorname{sinc}(i-k) \mathrm{e}^{j \pi(i-k)}, \sin (x)=\frac{\sin (\pi x)}{(\pi x)}$, and $W_{m}(k)=\sum_{n=0}^{N-1} w\left(n+N_{m}\right) e^{-j \frac{2 \pi}{N} n k}$. Besides $\delta_{i, k}=1$ with $\mathrm{i}=\mathrm{k}$ and $\delta_{i, k}=0$ with i $\neq$ k. So $Y_{m}(k)=X_{m}(k) H(k)+W_{m}(k)$ and sub-carriers are perfectly orthorgonal at the receiver.

\section{B. Restrictions of OFDM}

OFDM technique only operates well when the orthogonality of sub-carriers is still maintained. If the characteristic is not good, ISI and ICI will appear. They consist of CFO, SFO, TO (timing offset), PHN (phase noise), time-varying channel $[11,12]$.

\section{ALGORITHM COMBINING SYNCHRONIZATION AND CHANNEL ESTIMATION}

This section presents an algorithm combining synchronization and channel estimation using pilot for Burst-mode OFDM systems. The block diagram of the algorithm at the receiver is showed in Fig. 2.

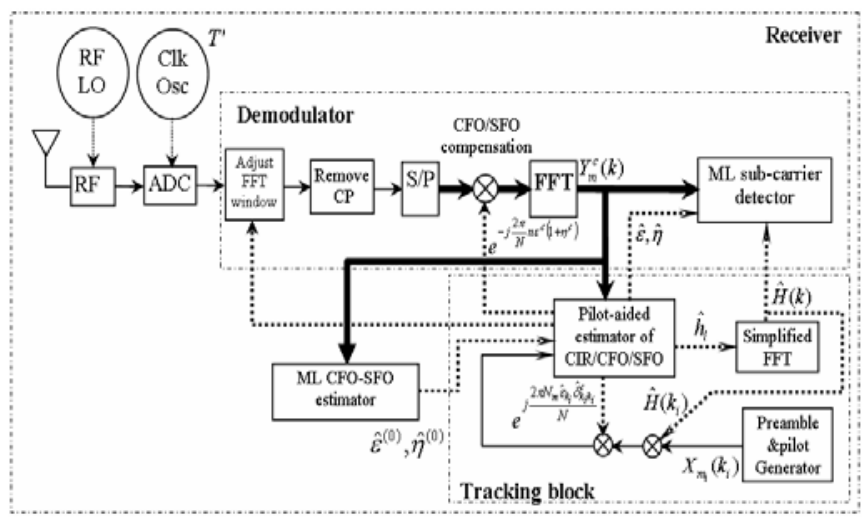

Figure 2: The receiver of Burst-mode OFDM utilizes the algorithm combining synchronization and channel estimation using pilot

In Fig. 2, Pilot-aided estimator of CIR (Channel Impulse Response)/CFO/SFO is the main block. This block utilizes RLS (Recursive Least-Squares) algorithm to estimate desired CIR, CFO and SFO values. The first value of the algorithm is taken from ML (Maximum-Likelihood) CFO-SFO estimator. After estimating CIR, SFO and CFO, these values are entered ML sub-carrier detector to detect transmitted signals.

The algorithm can be summarized as follows: with pilot tones of received signals in frequency, we build a cost function including parameters: CFO, SFO and CIR. The cost function is used to deploy the Recursive Least-Squares algorithm and tracking algorithm. The same recursive algorithms, the estimation method that uses RLS algorithm also needs some initial samples to converge. So, in the first, we use ML algorithm that 
relies on Preamble to estimate rough values of $\mathrm{CFO}$, SFO. These roughly estimated values are used to overcome large affects of ICI (caused by CFO, SFO), they are first values enhancing performance and converging speed of the algorithm.

\section{A. Acquisition phase}

This algorithm was represented with assumptions:

- A rough estimation algorithm to examine initial time of symbols is performed in preamble, so that the receiver initializes to sample at the range that is affected by ISI.

- To decrease affections of frequency offset helping the algorithm operate better, a rough frequency offset estimator is used.

Based on periodic construction of short preamble symbols, the solution for the rough timing estimator and the frequency offset estimator of carrier is AutoCorrelator. The auto-correlator is shown in Fig 3. $\mathrm{N}_{d}$ and $\mathrm{N}_{\text {avg }}$ is main parameters, $\mathrm{N}_{\mathrm{d}}$ is the delay value entered signal, while $\mathrm{N}_{\mathrm{avg}}$ is the long avarage of Moving Average filter.

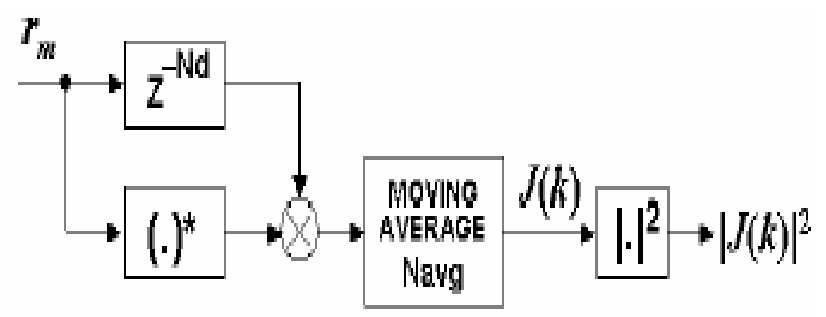

Figure 3: Block diagram of the Auto-Correlator

Using the signal in equation (1) for an OFDM frame, where symbols obey $U(t)$ impulse function, and assuming frames are transmitted to channel having AWGN noise, the received signal is given by:

$$
r(t)=s(t) \cdot e^{j 2 \pi \frac{\varepsilon}{T} t}+v(t)
$$

where $\varepsilon / T$ is frequency offset carrier, $v(t)$ is white noise obeying the Gauss distribution (zero mean). The signal in equation (5) samples at $1 / T_{\text {sam }}$. So, the received OFDM signal is given by:

$$
r(m)=s(m) e^{j 2 \pi \varepsilon \frac{\Delta f}{f_{\text {sam }}} m}+v(m)
$$

where $s(m)$ is initially transmitted OFDM signal, $\varepsilon$ is frequency offset carrier normalized, $\Delta f$ is the frequency interval of sub-carriers in OFDM signal and $f_{\text {sam }}$ is sampling frequency. $v(m)$ sequence shows white noise process having zero mean. According to IEEE 802.11a [1], $\Delta f=312.5 \mathrm{kHz}$ and $f_{\text {sam }}=20$ $\mathrm{MHz}$.

The signal in Fig. 3 can be expressed by:

$$
\begin{aligned}
& J(k)=\sum_{l=0}^{N_{a g}-1} r^{*}(l-k) r\left(l-k-N_{d}\right)
\end{aligned}
$$

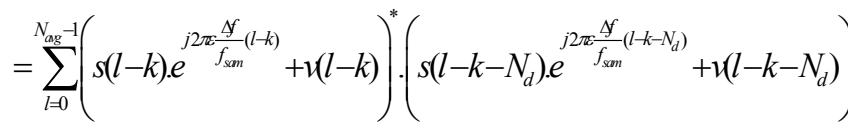

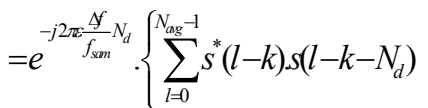

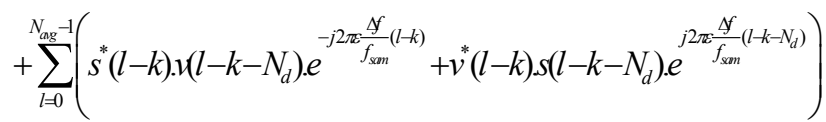

$$
\begin{aligned}
& \left.+\sum_{l=0}^{N_{a g}-1} v^{*}(l-k) \cdot v\left(l-k-N_{d}\right)\right\}
\end{aligned}
$$

Assuming that $s(m)$ uncorrelates with $v(m)$ noise, the two last components in equation (7) can be ignored as $N_{\text {avg }}$ is great enough, then:

$$
\begin{aligned}
J(k) & \approx e^{-j 2 \pi \varepsilon \frac{\Delta f}{f_{\text {sam }}} N_{d}} \cdot \sum_{l=0}^{N_{\text {avg }}-1} s^{*}(l-k) \cdot s\left(l-k-N_{d}\right) \\
& =e^{-j 2 \pi \varepsilon \frac{\Delta f}{f_{\text {sam }}} N_{d}} \cdot \sum_{l=0}^{N_{\text {avg }}-1}|s(l-k)|^{2},
\end{aligned}
$$

In this case, $s(m)$ is periodic with $N_{d}$ samples period, $s(m)=s\left(m-N_{d}\right)$. From equation (8), $J(k)$ phase only depends on $\varepsilon$, and $\varepsilon$ can be determined by:

$$
\varepsilon=\frac{f_{\text {sam }}}{2 \pi \cdot N_{d} . \Delta f} \tan ^{-1}\left(J^{*}(k)\right)
$$

As $\left(f_{s} / \Delta f\right)$ is a constant, estimation value of $\varepsilon$ only depends on $N_{d}$ in Auto-correlator. In IEEE 802.11a, from equation (9), relationship between estimation value $\varepsilon$ and $N_{d}$ is given by as Table 1 . 
Table 1: Estimation value $\varepsilon$ for different $N_{d}$ values

\begin{tabular}{|c|c|}
\hline$N_{d}$ & $|\varepsilon|_{\max }$ \\
\hline 16 & 2.0 \\
\hline 32 & 1.0 \\
\hline 48 & 0.66 \\
\hline 64 & 0.5 \\
\hline
\end{tabular}

\section{B. Decreasing ICI by compensating CFO-SFO}

By using $\mathrm{CFO}$ and $\mathrm{SFO}$ after rejecting $\mathrm{CP}, n^{\text {th }}$ sample in $m^{\text {th }}$ symbol of received signal in time domain can be shown:

$$
r_{m, n}^{c, n}=\frac{e^{j \frac{j \pi}{N}\left(N_{m}+n\right) \varepsilon_{\eta}}}{N} \sum_{k=-K / 2}^{K / 2-1} X_{m}(k) H(k) e^{j \frac{2 \pi k}{N} N(1+\eta \eta)} e^{\frac{2 \pi k k_{n}}{N} \eta N_{m}}+w_{m}\left(n+N_{m}\right)
$$

where $n=0,1, \ldots, N-1$ and $N_{m}=N_{g}+m\left(N+N_{g}\right), w_{m}\left(n+N_{m}\right)$ is noise with Gauss distribution that is complex numbers, its mean is zero and covariance is $\sigma^{2}$, $H(k)=\sum_{l=0}^{L-1} h_{l} e^{-j \frac{2 \pi k}{N} l}$ is channel response of the $\mathrm{k}^{\text {th }}$ subcarrier. To eliminate ISI completely, $\mathrm{CP}$ must be longer than excess delay of channel, L. CFO and SFO are normalized with sampling period $\mathrm{T}$ in the transmitter that has the order $\eta=\Delta T / T, \Delta T=T^{\prime}-T$, $\varepsilon=\Delta f N T=(\Delta f / f)(N T f)$ and $\varepsilon_{\eta}=(1+\eta) \varepsilon$. In practice, both of $\Delta \mathrm{T} / \mathrm{T}$ and $\Delta \mathrm{f} / \mathrm{f}$ are in acceptable interval, normally 10ppm (10E-6) or smaller. However, frequency carrier $f$ is often much more than sampling frequency $1 / T$, so $N T f$ coeffient can create great $\mathrm{CFO}(\varepsilon)$ and small $\mathrm{SFO}(\eta)<<1$. After FFT, the received sample in frequency domain is $Y_{m}(k)=\sum_{n=0}^{N-1} r_{m, n}^{\varepsilon, n} e^{-j \frac{2 \pi}{N} n k}$. From equation (9), we can show:

$$
\begin{aligned}
Y_{m}(k) & =\sum_{i=-K / 2}^{K / 2-1} X_{m}(i) H(i) e^{j \frac{2 \pi}{N} N_{m} \varepsilon_{i}}\left(\frac{1}{N} \sum_{n=0}^{N-1} \mathrm{e}^{j \frac{2 \pi}{N} n\left(\varepsilon_{i}+i-k\right)}\right)+W_{m}(k) \\
& =\sum_{i=-K / 2}^{K / 2-1} X_{m}(i) H(i) e^{j \frac{2 \pi}{N} N_{m} \varepsilon_{i}} \delta_{i, k}+W_{m}(k)
\end{aligned}
$$

where

$$
\begin{aligned}
& \delta_{i k}=\frac{1}{N} \sum_{n=0}^{N-1} \mathrm{e}^{j \frac{2 \pi}{N} n\left(\varepsilon_{i}+i-k\right)}=\frac{1}{N} \cdot \frac{1-\mathrm{e}^{j 2 \pi\left(\varepsilon_{i}+i-k\right)}}{1-\mathrm{e}^{j \frac{2 \pi}{N}\left(\varepsilon_{i}+i-k\right)}} \approx \operatorname{sinc}\left(\varepsilon_{i}+i-k\right) \mathrm{e}^{j \pi\left(\varepsilon_{i}+i-k\right)}, \\
& \varepsilon_{i}=i \eta+\varepsilon_{\eta}, \sin (x)=\frac{\sin (\pi x)}{(\pi x)}, W_{m}(k)=\sum_{n=0}^{N-1} w\left(n+N_{m}\right) e^{-j \frac{2 \pi}{N} n k}
\end{aligned}
$$

The compenent $\varepsilon_{i}=i \eta+\varepsilon_{\eta}$ need to be rejected to destroy ICI. On the other hand, to destroy we ICI, we need to compensate affections of CFO and SFO in carriers in frequency domain.

The above formula shows CFO and SFO which will create a rotation in time domain and a decrease as well as ICI in frequency domain. Decrease can be solved easily by compensating symbol-by-symbol. To reject ICI, detected symbols in frequency domain need to be known. Therefore, the best solution is the rotation in time domain to against ICI in frequency domain. After FFT, sub-carriers in frequency domain are:

$$
\begin{aligned}
& Y_{m}^{c}(k)=\sum_{n=0}^{N-1} r_{m, n}^{c} e^{-j \frac{2 \pi}{N} n k}=\sum_{n=0}^{N-1} r_{m, n}^{n, \varepsilon} e^{-j \frac{2 \pi}{N} n e_{n}^{c}} \cdot e^{-j \frac{2 \pi}{N} n k}
\end{aligned}
$$

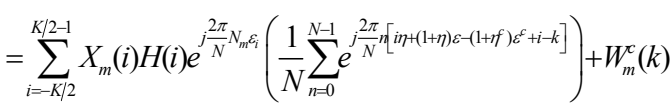

$$
\begin{aligned}
& =\sum_{i=-K / 2}^{K / 2-1} X_{m}(i) H(i) e^{j \frac{j \pi}{N} N_{m} \varepsilon_{i}} \delta_{i, k}^{c}+W_{m}^{c}(k)
\end{aligned}
$$

where

$$
W_{m}^{c}(k)=\sum_{n=0}^{N-1} w_{m}\left(n+N_{m}\right) e^{-j \frac{2 \pi}{N} n\left(1+\eta^{c}\right)_{c}^{c}} e^{-j \frac{2 \pi}{N} n k}
$$

and $\delta_{i, k}^{c}=\frac{1}{N} \sum_{n=0}^{N-1} e^{j \frac{2 \pi}{N} n\left[i \eta+(1+\eta) \varepsilon-\left(1+\eta^{c}\right) \varepsilon^{c}+i-k\right]}$.

So, after TD (Time Domain) CFO-SFO Compensation, ICI coefficient becomes:

$$
\delta_{i, k}^{c}=\frac{1}{N} \sum_{n=0}^{N-1} e^{j \frac{2 \pi}{N} n\left[i \eta+\varepsilon_{\eta}-\varepsilon_{\eta}^{c}+i-k\right]}
$$

From equation (13), by using TD CFO-SFO Compensation as perfect $\mathrm{CFO}$ and $\mathrm{SFO}\left(\varepsilon^{c}=\varepsilon\right.$ and $\eta^{c}=\eta$ ) estimations, ICI coefficient is given by:

$$
\delta_{i, k}^{c}=\frac{1}{N} \sum_{n=0}^{N-1} e^{j \frac{2 \pi}{N} n[i \eta+i-k]}
$$

Clearly, ICI coefficient is destroyed significantly. However, ICI still attends by in component. In fact, because of $\operatorname{SFO}(\eta)<<1$, ought to this noise should be ignored.

To examine effect of TD CFO-SFO Compensation block, defining ISR (ICI-to-signal ratio) by:

$$
I S R=\frac{P_{I C I}}{P_{s}}
$$

where 


$$
\begin{aligned}
& P_{I C I}=E\left(\left|\sum_{i=-K / 2}^{K / 2-1} X_{m}(i) H(i) e^{j \frac{2 \pi N_{m} \varepsilon_{i}}{N}} \delta_{i, k}^{c}\right|^{2}\right) \text { and } \\
& P_{s}=E\left(\left|X_{m}(k)\right|^{2}|H(k)|^{2}\left|\delta_{k, k}^{c}\right|^{2}\right) .
\end{aligned}
$$

After calculating, the result is:

$$
I S R=\left(\sum_{k=-K / 2}^{K / 2-1} \sum_{\substack{i=-K / 2 \\ i \neq k}}^{K / 2-1}\left|\delta_{i, k}^{c}\right|^{2}\right) / \sum_{k=-K / 2}^{K / 2-1}\left|\delta_{k, k}^{c}\right|^{2}
$$

\section{Combining CIR, CFO and SFO by using Pilot}

Basing on received sub-carriers in frequency domain, the algorithm using pilot tries to estimate CIR, CFO and SFO. RLS algorithm is used here to estimate CIR channel coefficients, CFO and SFO in frequency domain by minimizing the LS cost function. LS cost of $i^{\text {th }}$ pilot tone in OFDM symbols of a data packet is given by:

$$
C\left(\hat{\mathbf{h}}^{(i)}, \hat{\varepsilon}^{(i)}, \hat{\eta}^{(i)}\right)=\sum_{p=1}^{i} \lambda^{i-p}\left|e_{i, p}\right|^{2}
$$

where $\lambda$ is Forgetting Factor of RLS and

$\hat{\mathbf{h}}^{(i)}=\left[\hat{h}_{0}^{(i)}, \hat{h}_{1}^{(i)}, \ldots \hat{h}_{L-1}^{(i)}\right]^{T}, \quad \hat{H}^{(i)}\left(k_{p}\right)=\sum_{l=0}^{L-1} \hat{h}_{l}^{(i)} e^{-j \frac{2 \pi k_{p} l}{N}}$,

$e_{i, p}=Y_{m_{p}}^{c}\left(k_{p}\right)-X_{m_{p}}\left(k_{p}\right) \hat{H}^{(i)}\left(k_{p}\right) e^{j \frac{2 \pi}{N} N_{m} \hat{\varepsilon}_{k_{p}}^{(i)}} \hat{\delta}_{i, k_{p}, k_{p}}^{c}$,

$\hat{\varepsilon}_{k_{p}}^{(i)}=k_{p} \hat{\eta}^{(i)}+\left(1+\hat{\eta}^{(i)}\right) \hat{\varepsilon}^{(i)}$,

$\hat{\delta}_{i, k_{p}, k_{p}}^{c}=\frac{1}{N} \sum_{n=0}^{N-1} e^{j \frac{2 \pi}{N} n\left[k_{p} \hat{\eta}^{(i)}+\left(1+\hat{\eta}^{(i)}\right) \hat{\varepsilon}^{(i)}-\left(1+\eta^{c}\right) \varepsilon^{c}\right]}$,

$p=1, \ldots, i$ denotes $p^{\text {th }}$ pilot tone index in the set of $\mathrm{i}^{\text {th }}$ pilot tone used in RLS algorithm, and $X_{m_{p}}\left(k_{p}\right)$ is the value of $p^{\text {th }}$ pilot tone of the $k_{p}{ }^{\text {th }}$ sub-carrier of the $m_{p}{ }^{\text {th }}$ OFDM symbol at $\mathrm{p}^{\text {th }}$ time index in RLS algorithm. Note that all using tones are the $1^{\text {st }}$ pilot in preamble of a data packet.

Appearance of (CFO,SFO) synchronization error in received samples causes $e_{i, p}$ estimation error. This is an unlinear function of estimation parameters. This case can't use an adaptively linear algorithm to estimate coefficients. So, in order to use adaptively traditional algorithm, unlinear estimation errors $e_{i, p}$ need to be linearized according to estimation parameters by expansing first-class Taylor sequence.

$e_{i, p} \approx Y_{m_{p}}^{c}\left(k_{p}\right)-\left\{f\left(X_{m_{p}}\left(k_{p}\right), \hat{\boldsymbol{\omega}}_{i-1}\right)+\nabla f^{T}\left(X_{m_{p}}\left(k_{p}\right), \hat{\boldsymbol{\omega}}_{i-1}\right)\left(\hat{\boldsymbol{\omega}}_{i}-\hat{\boldsymbol{\omega}}_{i-1}\right)\right\}$

where

$f\left(X_{m_{p}}\left(k_{p}\right), \hat{\boldsymbol{\omega}}_{i-1}\right)=X_{m_{p}}\left(k_{p}\right) \hat{H}^{(i-1)}\left(k_{p}\right) e^{j \frac{j \pi}{N} N_{m} \hat{\varepsilon}_{k_{p}}^{(-1)}} \hat{\delta}_{i-1, k_{p}, k_{p}}^{c}$, and $\hat{\boldsymbol{\omega}}_{i}=\left[\hat{\omega}_{i, 0}, \hat{\omega}_{i, 1}, \ldots, \hat{\omega}_{i, L+1}\right]^{T}$ is a vector of size $(\mathrm{L}+2) \times 1$ that consists of estimation values of CIR, CFO and $\mathrm{SFO}$ at the $\mathrm{i}^{\text {th }}$ time of RLS algorithm, namely:

$\hat{\omega}_{i, l}=\hat{h}_{l}^{(i)}$, for $l=0,1, \ldots,(L-1), \hat{\omega}_{i, L}=\hat{\varepsilon}^{(i)}, \hat{\omega}_{i, L+1}=\hat{\eta}^{(i)}$.

Gradient Vector is calculated according to following:

$\nabla f\left(X_{m_{p}}\left(k_{p}\right), \hat{\boldsymbol{\omega}}_{i}\right)=\left[\frac{\partial f\left(X_{m_{p}}\left(k_{p}\right), \hat{\boldsymbol{\omega}}_{i}\right)}{\partial \hat{\boldsymbol{\omega}}_{i, 0}}, \ldots, \frac{\partial f\left(X_{m_{p}}\left(k_{p}\right), \hat{\boldsymbol{\omega}}_{i}\right)}{\partial \hat{\omega}_{i, L+1}}\right]^{T}$

where:

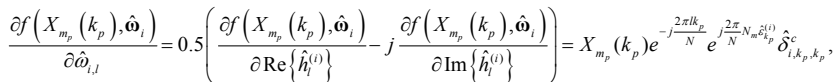

$$
\begin{aligned}
& \frac{\partial f\left(X_{m_{m}}\left(k_{p}\right), \hat{\omega}_{t}\right)}{\partial \hat{\omega}_{i, t}}=\left(1+\hat{\eta}^{(i)}\right) \Omega_{i, p}, \quad \frac{\partial f\left(X_{m_{m}}\left(k_{p}\right), \hat{\omega}_{i}\right)}{\partial \hat{\omega}_{t, t+1}}=\left(k_{p}+\hat{\varepsilon}^{(i)}\right) \Omega_{t, p}
\end{aligned}
$$

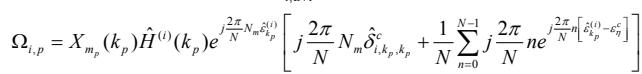

The algorithm combining estimations of CIR, CFO and SFO is based on RLS as showing in Fig 4.

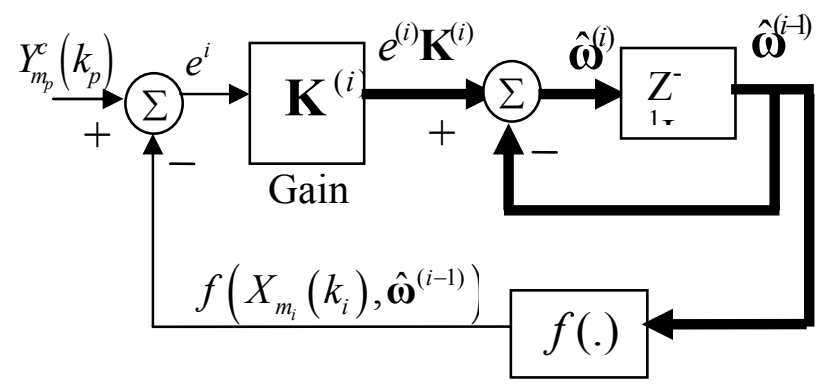

Figure 4: The block diagram of combination between synchronization and channel estimation using pilot

The $i^{\text {th }}$ estimated value is updated as follows:

$$
\hat{\boldsymbol{\omega}}^{(i)}=\hat{\boldsymbol{\omega}}^{(i-1)}+e^{(i)} \mathbf{K}^{(i)}
$$

where,

$$
\begin{aligned}
\mathbf{K}^{(i)} & =\frac{\mathbf{P}^{(i-1)} \cdot \nabla f^{*}\left(X_{m_{i}}\left(k_{i}\right), \hat{\boldsymbol{\omega}}^{(i-1)}\right)}{\lambda+\nabla f^{T} \cdot\left(X_{m_{i}}\left(k_{i}\right), \hat{\boldsymbol{\omega}}^{(i-1)}\right) \cdot \mathbf{P}^{(i-1)} \cdot \nabla f^{*}\left(X_{m_{i}}\left(k_{i}\right), \hat{\boldsymbol{\omega}}^{(i-1)}\right)} \\
e^{(i)} & =Y_{m_{i}}^{c}\left(k_{i}\right)-f\left(X_{m_{i}}\left(k_{i}\right), \hat{\boldsymbol{\omega}}^{(i-1)}\right) \quad \mathbf{v a ̀ ~}
\end{aligned}
$$




$$
\begin{gathered}
\mathbf{P}^{(i)}=\frac{1}{\lambda}\left(\mathbf{P}^{(i-1)}-\mathbf{K}^{(i)} \nabla f^{T}\left(X_{m_{i}}\left(k_{i}\right), \hat{\boldsymbol{\omega}}^{(i-1)}\right) \mathbf{P}^{(i-1)}\right) \\
f\left(X_{m_{p}}\left(k_{p}\right), \hat{\boldsymbol{\omega}}_{i-1}\right)=X_{m_{p}}\left(k_{p}\right) \hat{H}^{(i-1)}\left(k_{p}\right) e^{j \frac{2 \pi}{N} N_{m} \hat{\hat{k}}_{k_{p}}^{(i-1)}} \hat{\delta}_{i-1, k_{p}, k_{p}}^{c},
\end{gathered}
$$

and $\nabla f\left(X_{m_{p}}\left(k_{p}\right), \hat{\boldsymbol{\omega}}_{i}\right)$ is calculated from equation (19).

The algorithm based on RLS helps estimating fastly and reducing errors when comparing with low stableness [10].

\section{The rough estimation $\mathrm{CFO}, \mathrm{SFO}$}

As other estimation algorithms, the estimation by using RLS also requires initial values of estimation parameters suitably to converge. ML algorithm is used to find rough estimation values of $\mathrm{CFO}$ and SFO. These two rough values are used as initial values of RLS algorithm.

Basing on usage sub-carriers of received signal in frequency domain corresponding to two long training symbols, ML cost function is defined as:

$$
f(\varepsilon, \eta)=\sum_{k \in I_{p}}\left|Y(k)-e^{j \frac{2 \pi N_{s}}{N}[k \eta+\varepsilon(1+\eta)]}\right|^{2}
$$

where $I_{p}$ is sub-carriers indexes's set of pilot tones in Preamble.

So, as rejecting CIR, rough estimation values of $\mathrm{CFO}$ and $\mathrm{SFO}$ can be calculated:

$$
\hat{\varepsilon}, \hat{\eta}=\underset{\varepsilon, \eta}{\arg \min } \sum_{k \in I_{p}} \mid Y(k)-e^{\left.j \frac{2 \pi N_{s}}{N}[k \eta+\varepsilon(1+\eta)]\right|^{2}}
$$

\section{E. The ML Sub-Carrier Detector}

In the OFDM receiver (Fig. 2), CIR, CFO and SFO are updated for each symbol. They are input for ML sub-carrier detector, while tracking block updates $\mathrm{CIR}, \mathrm{CFO}, \mathrm{SFO}$ in each sample. Besides, because the number of CIR index is less than FFT size, a simple FFT block is used to synthesise transmission function that is used in sub-carrier detector for demodulations and correction signals transmitted in tracking block.

After FFT block, ML method is used to detect received signals. A symbol in frequency domain is given by:

$$
\hat{X}_{m}(k)=\underset{X_{m}(k)}{\arg \min }\left\{\left|Y_{m}^{c}(k)-X_{m}(k) \hat{H}(k) e^{j \frac{2 \pi}{N} N_{m} \hat{\varepsilon}_{k}} \hat{\delta}_{k k}^{c}\right|^{2}\right\}
$$

\section{SIMULATION RESULTS}

The paper use Matlab 7.0 for simulations to evaluate the algorithm. OFDM parameters are selected to be the same with IEEE 802.11a standard [1] as follows: 52 sub-carriers (48 for data and 4 for pilot that is the same power), CP interval is 16 samples, FFT size is 64 .

BER (Bit Error Rate) is examined to evaluate the accuracy of the algorithm. Therefore, BER is simulated with changes of SNR (Signal-to-Noise Ratio), CFO and SFO.

The results of BER vs. SNR are showed in Fig. 5, whre the channel is AWGN and Rayleigh fading, using 16-QAM và 64-QAM modulation. Furthermore, the results also show with ideal instance, in this case, channel estimation and synchronization is perfect $(\mathrm{SFO}=\mathrm{CFO}=0)$.

The results show that the algorithm performs well for different modulations. A, B, E and F lines in Fig $5 \mathrm{a}$ and $5 \mathrm{~b}$ show that the results according to theory and ideal instance are very similar in both AWGN and multi-path Raleigh channel. To examine the algorithm accurately, the instance that has $\mathrm{CFO}=0.212$ and $\mathrm{SFO}=112 \mathrm{E}-6$ in Rayleigh multipath fading channel with changes in some other parametes is simulated.

For the case that has no decreasing ICI (not compensating $\mathrm{CFO}$ and SFO). The D line in Fig 5a and $5 \mathrm{~b}$ shows that though ML CFO-SFO estimator is still used, BER is still very high (BER is 1E-1 for 16QAM and 2E-1 for 64-QAM).

In the case that uses ML CFO-SFO estimator and ICI compensation concurrently, the algorithm shows that it operates well, the differences between it and the ideal instance are very little in both AWGN (G line) and multi-path Rayleigh fading (C line). 
Therefore, using ML CFO-SFO estimator and ICI compensation is very necessary in the algorithm.

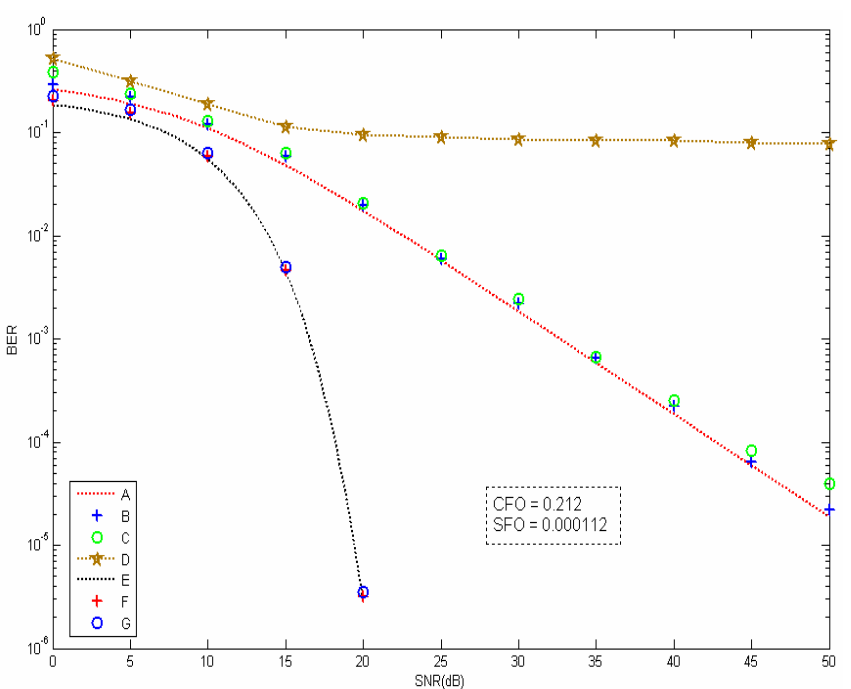

(a)

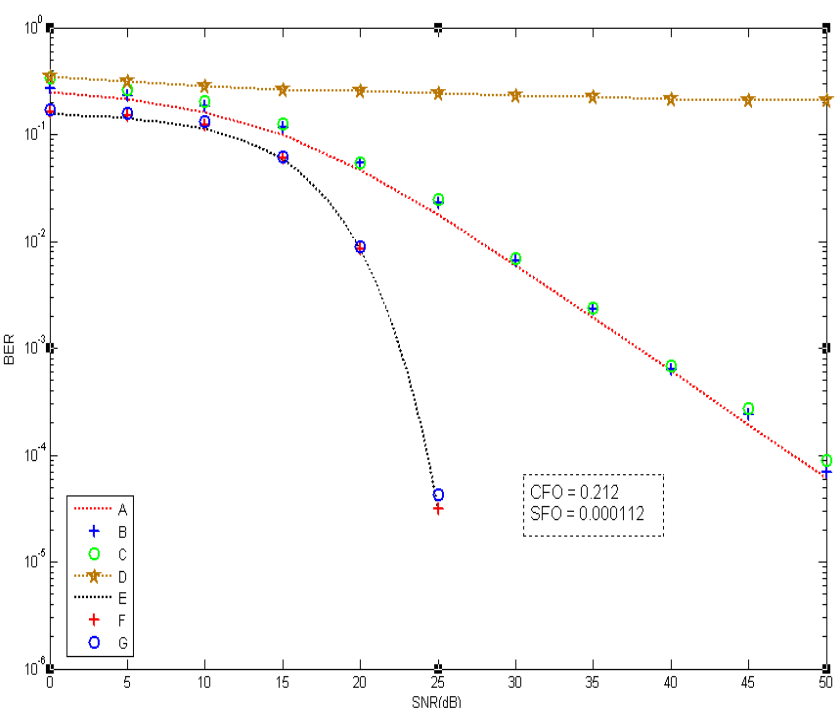

(b)

Figure 5: BER vs. SNR by: a) using 16-QAM modulation and b) using 64-QAM modulation. $A$ - BER according to theory, channel is Rayleigh; $B$ - Ideal synchronization and channel estimation, channel is Rayleigh; $C$ - Using concurrently synchronization and estimation, rough estimator and compensation CFO-SFO, channel is Rayleigh; $D$ - Using concurrently synchronization, estimation and rough estimator, not using CFO-SFO,

channel is Rayleigh; E-BER according to theory, channel is AWGN; F-Ideal synchronization and channel estimation, channel is AWGN; G - Using concurrently synchronization and estimation, rough estimator and compensation CFO-SFO, channel is AWGN

\section{CONCLUSION}

Results showed that the algorithm is good. Its results are close to the ideal (in the case channel estimation and synchronization are perfect) in AWGN and Rayleigh channel. Besides, results have also showed that the algorithm performs well for different modulations.

Data types of variables in the rough CFO-SFO estimator were surveyed. However, these values still unoptimized (for instance, the size of variables can be decreased). Therefore, detail studies about data types are necessary.

In OFDM systems, at practical receivers, beside $\mathrm{CFO}, \mathrm{SFO}$, an element causing restrictions to systems that can't be ignored is PHN. Our works in future will examine an algorithm combining channel estimation, CFO, SFO and PHN.

\section{REFERENCES}

[1] IEEE Computer Society, IEEE Std 802.11a-1999, Dec 1999.

[2] Y. Yao and G. B. Giannakis, "Blind carrier frequency offset estimation in SISO, MIMO, and multiuser OFDM systems," IEEE Trans. Commun., Vol. 53, N. 1, Jan. 2005, pp. 173-183.

[3] P. Y. Tsai, H-Y Kang, and T-D. Chiueh, "Joint weighted least-squares estimation of frequency and timing offset for OFDM systems over fading channels," IEEE Trans. Veh. Technol., Vol. 54, Issue 1, Jan. 2005, pp. 211-223.

[4] C. Oberli, Daneshrad, B., "Maximum likelihood tracking algorithms for MIMO-OFDM," IEEE ICC'04, June 2004, pp. 2468-2472.

[5] M. Shin, H. Lee, and C. Lee, "Enhanced channelestimation technique for MIMO-OFDM systems," IEEE Trans. Veh. Technol., Vol. 53, N. 1, Jan. 2004, pp.261-265.

[6] I. Barhumi, G. Leus, and Moonen, "Optimal training design for MIMO OFDM systems in mobile wireless channels," IEEE Trans. Signal Process., Vol. 51, N. 6, June 2003, pp. 1615-1624.

[7] J-H. Lee, J. C. Han, and S-C. Kim, "Joint carrier frequency synchronization and channel estimation for 
OFDM systems via the EM algorithm," IEEE Trans. Veh. Technol., Vol. 55, No. 1, Jan. 2006, pp. 167-172.

[8] D. D. Lin, R. A. Pacheco, T. J. Lim, and D. Hatzinakos, "Joint estimation of channel response, frequency offset, and phase noise in OFDM," IEEE Trans. on Signal Process., Vol. 54, Sept. 2006, pp. 3542-3554.

[9] S. Gault, W. Hachem, and P. Ciblat, "Joint sampling clock offset and channel estimation for OFDM signals: Cramer-Rao bound and algorithms," IEEE Trans. on Signal Process., Vol. 54, May 2006, pp. 1875-1885.

[10] S. B.Weinstein and P. M. Ebert, "Data transmission by frequency division multiplexing using the discrete fourier transform," IEEE Trans. Commun. Tech., Vol. COM-19, Oct. 1971.

[11] S.Wu and Y. Bar-Ness, "OFDM channel estimation in the presence of frequency offset and phase noise," in Proc. IEEE Int. Conf. Communications (ICC) 2003, Vol. 5, May 2003, pp. 3366-3370.

[12]D. D. Lin, R. A. Pacheco, T. J. Lim, and D. Hatzinakos, "Optimal OFDM channel estimation with carrier frequency offset and phase noise," in Proc. IEEE Wireless Communications Networking Conf.(WCNC) 2006, Apr. 2006.

\section{AUTHORS' BIOGRAPHIES}

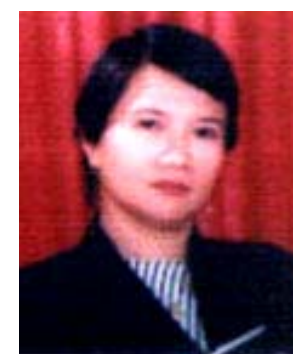

Pham Hong Lien received PhD. in Information Technology at the University of Technology Slovakia, 1993. She has been Assoc.Prof. since 2006.

Her research interests are Telecom \& Computer Network.

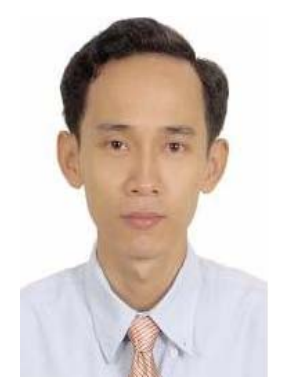

Nguyen Duy Lai received engineering bachelor in Electronics Technology 2002 and MSc at the HCM City University of Technology, 2009. Major research interests: Electronics and Telecomunications 